\title{
Norois
}

Environnement, aménagement, société

$240 \mid 2016$

Aménagement foncier, risque côtier, évolution des fleuves

\section{Le tableau politique de la France de l'Ouest. 100 ans après, héritages et postérités, André Siegfried}

\section{Christian Le Bart}

\section{OpenEdition}

\section{Journals}

Édition électronique

URL : http://journals.openedition.org/norois/5970

DOI : $10.4000 /$ norois. 5970

ISBN : 978-2-7535-5362-0

ISSN : $1760-8546$

\section{Éditeur}

Presses universitaires de Rennes

\section{Édition imprimée}

Date de publication : 30 novembre 2016

Pagination : 104-107

ISBN : 978-2-7535-5356-9

ISSN : 0029-182X

\section{Référence électronique}

Christian Le Bart, "Le tableau politique de la France de l'Ouest. 100 ans après, héritages et postérités, André Siegfried », Norois [En ligne], 240 | 2016, mis en ligne le 30 novembre 2016, consulté le 23 septembre 2020. URL : http://journals.openedition.org/norois/5970 ; DOI : https://doi.org/10.4000/ norois. 5970 


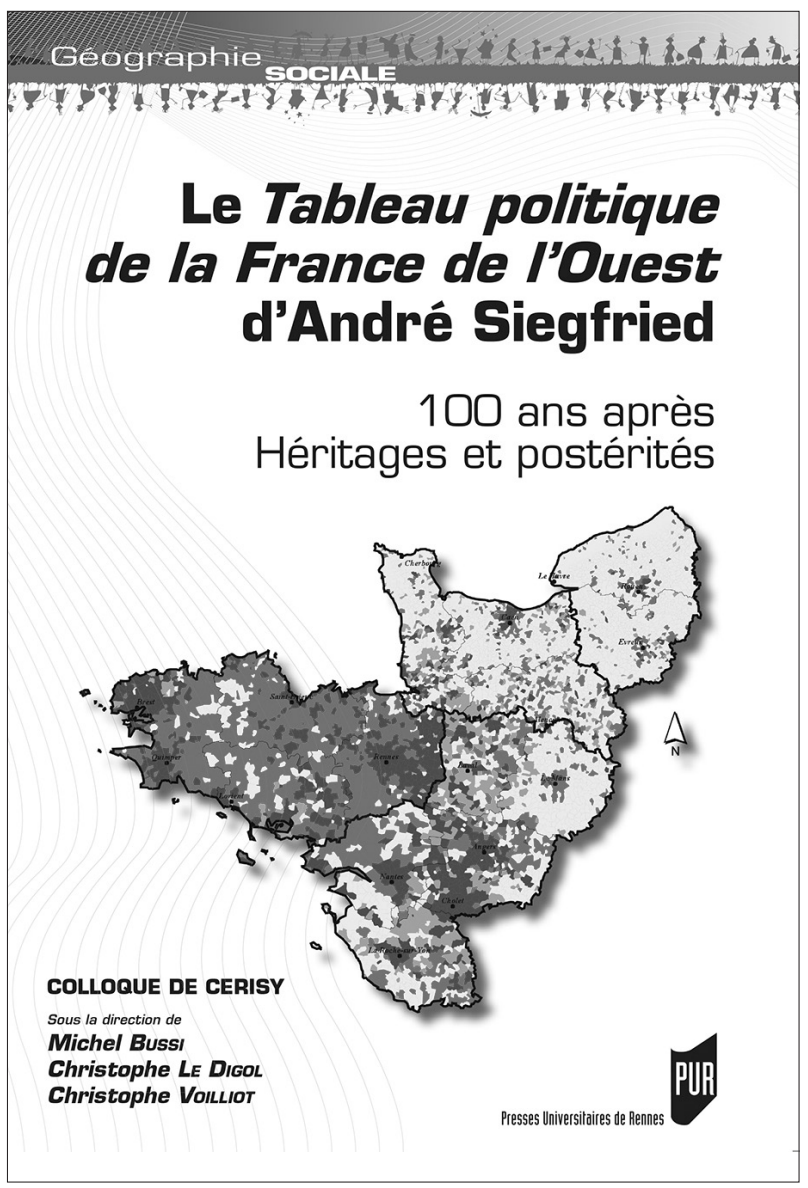

rale. Alain Garrigou revient sur l'épisode matriciel du double échec face au richissime aristocrate Boni de Castellanne (Basses-Alpes, 1902-1903). Il note avec ironie : "André Siegfried avait découvert la stabilité électorale dans les Basses-Alpes» (p. 38). Évoquant de même l'« entremêlement fonctionnel entre l'expérience personnelle accumulée au cours de deux campagnes législatives et la recherche savante aboutissant à la rédaction d'un ouvrage scientifique »(p. 19), André-Louis Sanguin restitue la carrière académique d'André Siegfried. À l'École Libre des Sciences Politiques, puis au Collège de France, celui-ci était d'abord considéré comme spécialiste des pays anglo-saxons, comme l'indiquent aussi bien la liste de ses enseignements que celle de ses publications (mentionnons quand même un retour à la sociologie électorale avec une Géographie électorale de l'Ardèche en 1949). Publié à compte d'auteur, mal vendu, pas traduit, le Tableau... sera redécouvert tardivement dans les années 1970 et surtout 1980, en particulier par un François Goguel désireux d'inscrire la sociologie électorale au cœur d'une science politique en pleine gestation. Côté géographes, comme le montrent Hermanvan der Wusten et Virginie Mamadouh, l'accueil est tiède et tardif malgré un compte-rendu positif de Vidal de la Blache. Sans doute Siegfried fut-il davantage remarqué aux États-Unis où il se rendait régulièrement, et où il pouvait apparaître comme pionnier (les auteurs mentionnent quelques précédents américains et norvégiens en matière de sociologie électorale). Cette discrétion n'empêche aucunement la consécration institutionnelle d'André Siegfried, dont Mathilde Sempé rappelle qu'il fut membre de l'Académie des sciences morales et politiques (1932) et de l'Académie française (1944). Sa position d'« outsider » dans le champ de la géographie, sa relation en demi-teinte aux historiens (Christophe Voilliot évoque la figure de Louis Chevalier, héritier finalement bien infidèle), tranchent avec de fortes positions institutionnelles au sein de la Fondation nationale des sciences politique, à l'ENA, à l'IEP de Paris... Souligné par plusieurs auteurs, et en particulier par Christophe Le Digol qui se demande « comment un auteur devient le père fondateur d'une discipline» (p. 119), son rôle (ou peut-être plutôt son enrôlement) dans la naissance de la science politique le consacre, et la Tableau... avec lui, comme père fondateur.

Les considérations biographiques et institutionnelles sont centrales, mais elles ne doivent pas masquer la nécessité de faire retour sur l'œuvre. Du côté des géographes, Michel Bussi revient sur le rapport distant de sa discipline à l'égard du travail de Siegfried. Le scepticisme de Vidal de la Blache a son origine dans une épistémologie jugée trop déterministe, trop intuitive. Ces reproches sont-ils fondés? Siegfried a le mérite de varier les approches, il étudie la presse, lit les professions de foi, il effectue des voyages d'étude sur le terrain... Il tente de marier démarche écologique et analyse psychologique, loin donc de la caricaturale alternative calcaire/granit à laquelle on le réduit trop souvent. Nul doute en revanche que le Tableau... ait vieilli s'agissant du recours, certes banal à l'époque mais irrecevable aujourd'hui, aux trop fameux tempéraments régionaux : "vendéen passionné », « personnalité fière des bretons bretonnants ", " mol angevin » (cité par Michel Bussi p. 99)... les arguments ethniques sonnent comme autant d'aveux d'im- 
puissance à expliquer des singularités locales qui se dérobent à l'analyse. Toujours côté géographes, Pascal Buléon dresse le même bilan : Siegfried est désireux de repérer des corrélations entre variables et de les formuler aussi clairement que possible ${ }^{1}$, il est prêt aussi à reconnaître que la concordance n'est qu'exceptionnelle et à combiner les variables. Comme son collègue, Pascal Buléon revient sur le déterminisme ethnique pour regretter que Siegfried ait davantage sollicité le mythe que l'histoire effective des mentalités et des formations socio-spatiales (expression empruntée à Guy Di Méo). Il insiste enfin sur les prolongements, certes parfois critiques, de l'œuvre de Siegfried : Paul Bois, Emmanuel Todd et Hervé Le Bras, Jean-Jacques Mounier l'ont corrigé, enrichi, mais aussi confirmé dans un contexte de relative inertie de la géopolitique de l'ouest (faibles migrations).

Au-delà de cette critique, il est clair que Siegfried fonde avec beaucoup de modernité l'approche écologique du vote. Côté science politique, Nathalie Dompnier relit Siegfried en mettant l'accent sur la façon dont l'indépendance de l'électeur est plus ou moins déterminée. Observant que Siegfried oscille entre approche macro-sociologique et approche relationnelle, elle distingue de la part des autorités sociales (patrons, propriétaires, administration, clergé) les stratégies de pression et les stratégies d'influence. Elle conclut que le passage de l'influence à la pression marque paradoxalement l'autonomisation du politique, puisque c'est l'exercice-même de celle-ci qui nourrit l'activité politique.

Il reste alors à prolonger Siegfried pour mesurer les changements de la carte électorale de l'ouest en un siècle. Pour les géographes, Jean Renard étudie sur un siècle le canton de Talmont en Vendée, exemple idéal-typique de discontinuité géologique entre Massif armoricain et plaine calcaire du Poitou (et de fort contraste politique). Il n'observe que peu de changements jusqu'aux années 1950, avant que l'ancienne frontière ne se brouille sous l'effet du tourisme, du déclin de l'agriculture, et de la périurbanisation. Jerome Prugneau et Emmanuel Bioteau tentent pour leur part d'évaluer ce qui demeure de la frontière constituée par une rivière (le Layon)

1. Ainsi dans un énoncé comme : « la géologie commandera le mode de peuplement en même temps que le mode d'exploitation, et par là, réagissant sur le mode de propriété et sur les rapports des classes entre elles, elle finira par avoir une répercussion sur la vie politique elle-même » (cité par Pascal Buléon, p. 132).
« transition entre l'Anjou sous influence angevine et l'aire vendéenne» (p. 220). Les auteurs interrogent au final ce qu'ils appellent la « discontinuité vécue» (p. 220).

Quid des villes? Michel Bussi propose une seconde contribution qui pose la nécessité d'une géographie électorale urbaine largement négligée par Siegfried. C'est par les villes que l'ouest glisse à gauche (municipales de 1977). Et l'auteur de proposer une typologie des villes mettant en relation profil électoral et caractéristiques socioéconomiques. Même ambition chez Jean Rivière, attentif à la sociologie des bureaux de vote et des quartiers (beaux quartiers orientés à droite, quartiers gentrifiés favorables aux gauches socialistes et écologistes, quartiers populaires caractérisés par une tendance au retrait du jeu électoral). La perspective urbaine est également dominante chez Danielle Rapetti, qui montre comment la région nantaise a évolué vers la gauche. Dans un contexte où la résidence apparaît moins liée à la profession et davantage au prix de l'immobilier, l'évolution électorale peut être saisie à partir de la variable revenu (évaluée à partir de l'IRPP).

Mentionnons enfin la contribution de Gilles Van Hamme, Christian Vandermotten, et Pablo Medina Lockhart, qui tentent, là encore cartographie à l'appui, de corréler à l'échelle européenne vote social-démocrate et par exemple classe ouvrière, ou bien vote écologiste et prospérité économique. Ces auteurs concluent que les continuités politiques sont moins dues à l'immobilité des structures sociales qu'à la stabilité des dispositifs d'encadrement que ces structures sociales ont fait naître et qui peuvent leur survivre. Ainsi seront centrales des variables comme le déclin des PC et de l'encadrement syndical, ou comme l'effondrement de la Démocratie chrétienne en Italie.

Côté politistes, les auteurs s'accordent sur la nécessité de dépasser la cartographie. Cherchant à mesurer le poids de la variable religieuse (dont il rappelle la centralité dans l'œuvre de Siegfried) Claude Dargent nuance la thèse de la sécularisation. Si on s'appuie sur les enquêtes post-électorales, on peut constater la force persistante du lien entre catholicisme et vote à droite. Certes les catholiques sont moins nombreux, certes ils sont dispersés (ce qui rend l'approche cartographique moins pertinente), mais ils demeurent électoralement typés. 
Effacement du déterminisme écologique au sens de Siegfried? Non, car on observe que « les catholiques d'une région très catholique votent plus à droite que leurs coreligionnaires des pays de mission » (p. 237). Ce que Claude Dargent appelle l'« amplification contextuelle» (p. 237).

Dans une contribution qui s'éloigne plus franchement encore d'André Siegfried, Jacques Le Bohec plaide pour une analyse fine des configurations spécifiques à chaque scrutin et à chaque territoire. Il revient sur la candidature de Jean-Marie Le Pen à Auray en 1983, à l'occasion d'une législative partielle occasionnée par la démission de Christian Bonnet. Les $12 \%$ obtenus au premier tour sont-ils imputables à l'" enfant du pays »? La crise de la pêche et de l'ostréiculture, le déclassement face à l'arrivée de riches retraités, sont des variables au moins aussi pertinentes. Et l'auteur d'évoquer une possible « homologie entre la situation de l'électeur dans son champ social de référence et celle du candidat dans le champ politique» (p. 246). Plus classique est l'approche de François Prigent qui, en historien du politique, revient sur les étapes qui ont marqué le glissement à gauche de la Bretagne. Activisme des chrétiens de gauche, implantation du PSU comme véritable laboratoire politique, événements fondateurs (grève du Joint français)... Le processus qui relie les victoires municipales de 1977 à la quasi hégémonie socialiste de 2014 n'a pourtant rien d'inexorable : le tarissement des réseaux militants fossilisés autour des leaders historiques (Edmond Hervé à Rennes) interroge la longue durée électorale chère à Siegfried...

Concluons sur un paradoxe remarquablement mis en avant dans ce livre collectif. Siegfried est célébré comme père fondateur d'une science politique qui, même dans sa dimension électorale, n'a de cesse de le dépasser en promouvant d'autres approches (sondages d'opinion, monographies ethnographiques...); tandis que la géographie électorale, moins disposée au fétichisme de l'œuvre fondatrice, continue à tirer des enseignements à partir de l'outil cartographique et à faire travailler le déterminisme écologique. Fortune paradoxale pour celui qui, comme le note fort justement Claude Dargent, « [traitait] les élections comme un fait social, ce que Durkheim luimême n’avait pas osé faire » (p. 224).

\section{Christian Le BarT Université Rennes 1}

